\title{
The Companion Dog as a Model for the Longevity Dividend
}

\author{
Kate E. Creevy ${ }^{1}$, Steven N. Austad ${ }^{2}$, Jessica M. Hoffman ${ }^{3}$, Dan G. O'Neill ${ }^{4}$, \\ and Daniel E.L. Promislow ${ }^{5}$ \\ ${ }^{1}$ Department of Small Animal Medicine and Surgery, College of Veterinary Medicine, \\ University of Georgia, Athens, Georgia 30602 \\ ${ }^{2}$ Department of Biology, University of Alabama at Birmingham, Birmingham, Alabama 35294 \\ ${ }^{3}$ Department of Genetics, University of Georgia, Athens, Georgia 30602 \\ ${ }^{4}$ Veterinary Epidemiology, Economics and Public Health, The Royal Veterinary College, \\ Hatfield, Herts AL9 7TA, United Kingdom \\ ${ }^{5}$ Departments of Pathology and Biology, University of Washington, Seattle, Washington 98195 \\ Correspondence: promislo@uw.edu
}

\begin{abstract}
The companion dog is the most phenotypically diverse species on the planet. This enormous variability between breeds extends not only to morphology and behavior but also to longevity and the disorders that affect dogs. There are remarkable overlaps and similarities between the human and canine species. Dogs closely share our human environment, including its many risk factors, and the veterinary infrastructure to manage health in dogs is second only to the medical infrastructure for humans. Distinct breed-based health profiles, along with their well-developed health record system and high overlap with the human environment, make the companion dog an exceptional model to improve understanding of the physiological, social, and economic impacts of the longevity dividend (LD). In this review, we describe what is already known about age-specific patterns of morbidity and mortality in companion dogs, and then explore whether this existing evidence supports the LD. We also discuss some potential limitations to using dogs as models of aging, including the fact that many dogs are euthanized before they have lived out their natural life span. Overall, we conclude that the companion dog offers high potential as a model system that will enable deeper research into the LD than is otherwise possible.
\end{abstract}

n a 2006 paper in The Scientist, Olshansky and colleagues introduced the notion of the longevity dividend (LD), which is defined as the social, economic, and health bonuses for both individuals and populations that accrue as a result of medical interventions to slow the rate of human aging (Olshansky et al. 2006). These investigators posit that, although permanently curing one or two chronic age-related diseases (e.g., heart disease, cancer) might lengthen both life span and health span a bit, a much more potent effect would result from slowing the underlying aging processes, which would reduce the risk and/or delay the age of onset

Editors: S. Jay Olshansky, George M. Martin, and James L. Kirkland

Additional Perspectives on Aging available at www.perspectivesinmedicine.org

Copyright (C) 2016 Cold Spring Harbor Laboratory Press; all rights reserved; doi: 10.1101/cshperspect.a026633

Cite this article as Cold Spring Harb Perspect Med 2016;6:a026633 
K.E. Creevy et al.

for most or all age-related maladies. These maladies include fatal and disabling diseases but also include nonfatal conditions, such as joint pain, hearing loss, dementia, or muscle weakness, which can degrade the quality of life.

The LD hypothesis rests on two key assumptions, one pathophysiological and the other demographic. First, the LD hypothesis assumes that if we ameliorate the underlying biological processes that drive aging, then necessarily we will reduce the frequency or delay the onset of most or all age-related disorders. Second, if we succeed in retarding the rate of aging, the period of debility toward the end of life will be compressed or at least maintained rather than lengthened. That is, we will extend the healthy phase of life without also extending the unhealthy phase.

Empirical evidence supporting both of these assumptions is mixed. In model laboratory species, interventions that increase longevity have been shown to retard some age-related functional declines, but to exacerbate others. For instance, with dietary restrictions (DRs) in laboratory mice, the poster child for extended health span studies, many aspects of health are in fact extended (Weindruch and Walford 1988). However, there are also some health downsides, such as increased susceptibility to some infectious diseases (Gardner 2005; Goldberg et al. 2015) and slowed wound healing throughout life (Reiser et al. 1995), not just near its end. Also, many genetic and environmental alterations that extend life span are associated with decreased fertility (Austad 2014). Although compromised fertility might not be directly related to mortality, it is certainly an important measure of the functional limitations of aging that Fries (1980) discussed in his classic paper on the compression of morbidity. A recent study in the laboratory nematode, Caenorhabditis elegans, was really the first to attempt to define worm health comprehensively and found while some-but by no means all-of four common worm longevity mutations increased the period of healthy life, all of these mutations also increased the duration of unhealthy life (Bansal et al. 2015).

Even what we know about humans, whose life spans have nearly doubled in the past
180 years (Oeppen and Vaupel 2002), is complicated. Mortality in general, and especially among the elderly, has continued to decline in virtually all developed countries, including the United States. This decline has been accompanied by prolonged health and morbidity compression in a number of countries (Thatcher et al. 2010). However, some indicators of laterlife health, such as functional mobility, appear to be have worsened since the last years of the 20th century (Crimmins and Beltran-Sanchez 2011), and this is a particularly worrisome trend among cohorts of the "near elderly" (Freedman et al. 2013). Additionally, in global surveys over the past 20 years, life expectancy at birth appears to be increasing more rapidly than healthy life expectancy, suggesting that more unhealthy years of life may be in our future (Salomon et al. 2012).

These debates make clear that to fully appreciate the consequences of extended longevity, both beneficial and deleterious, we need to develop a comprehensive understanding of the underlying causes of mortality, of how risks associated with these causes change with age, and of the impact on health span of reducing or eliminating cause-specific mortality as contrasted with an overall slowing of the rate of aging.

These challenges are addressed throughout this collection, including discussion of the potential to study the LD in common laboratory model species, such as the nematode worm C. elegans, the fruit fly Drosophila melanogaster, and the house mouse Mus musculus. Although these model organisms have obvious strengths for providing insight into the dynamics of life span versus health span, they also suffer from various limitations. Especially in worms and flies, we have a limited understanding of the underlying causes of death and our measures of health in these species are also not well developed, tending to focus on traits such as stress resistance, locomotion, mating behavior, or feeding rates (Burger and Promislow 2006; Bansal et al. 2015). In mice, the limitations are somewhat different. Although mouse postmortem histopathology is quite sophisticated, determination of health status in living animals is crude and still being developed. Currently, 
mouse health assays mainly use tools that were developed to assess genetically induced diseases. Mice that are commonly used in research also die of a restricted range of causes that do not reflect common causes of human deaths. For instance, mice do not spontaneously develop atherosclerosis and seldom develop neurodegenerative diseases. Even the common cancers that spontaneously develop in mice differ considerably from the common cancers in people. Mouse cancers are primarily lymphomas and sarcomas rather than the carcinomas that dominate the cancer spectrum in aging humans (Chang 2005).

In this review, we explore the possibility that companion dogs might offer an excellent model to study the potential of the LD to increase human health span. Dogs offer a number of strengths relative to existing animal model systems. First, our understanding of age-specific morbidity and mortality in companion dogs, and the medical infrastructure to treat their diseases, are second only to that of humans. Second, variation between breeds in their frequency of different age-specific diseases points to a strong genetic component. Third, the complex nature of dog behavior and physiology, both healthy and pathological, and the fact that these companion animals share our homes and our daily routines, suggests that we can develop meaningful measures of health span in companion dogs that will also be relevant in humans. Fourth, the shorter average life spans of dogs, $\sim 12$ years (O'Neill et al. 2013) compared with humans means that we can obtain results much faster, enabling speedier recommendations for improved human health. And, finally, there is value to understanding aging in dogs not only as a model for humans, but also because dogs are enormously valued as companions and owners want their older dogs to be healthy.

\section{DOGS, AGING, AND THE LONGEVITY DIVIDEND}

Dogs have been living in association with humans for at least 15,000 years and likely were domesticated multiple times across Eurasia from groups of wolves that foraged on refuse
The Dog as a Model for the Longevity Dividend

from human encampments (Larson et al. 2012; Freedman et al. 2014). Millennia of coevolution followed, such that humans became particularly sensitive to dogs' postural communication and vice versa (Miklosi and Soproni 2006; Kaminski et al. 2012), and dogs have been used in a vast array of human activities from hunting to herding to bomb, drug, and cadaver detection to assistance for the disabled to simple companionship.

\section{Variability}

Thanks to selective breeding for particular purposes and the aesthetics of breed development, dogs stand out as the most phenotypically variable mammal on earth. From even the most cursory observation of dogs playing in the park, the breadth of both behavioral and morphological diversity within this species is striking. Equally impressive is the demographic variation that we see between different dog breeds, with life expectancy varying from $\sim 6$ years to 16 years (Egenvall et al. 2005a; Adams et al. 2010; O’Neill et al. 2013).

This variation points to a strong genetic basis not only for visible phenotypes, but also for longevity and disease predisposition. Moreover, breed-specific risks of morbidity and mortality are strongly associated with breed-specific differences in size (Li et al. 1996; Greer et al. 2007; Kraus et al. 2013). Unlike the pattern across mammalian species, in which larger species tend to live longer (Austad and Fischer 1991; Promislow 1993), within the domesticated dog, the smaller breeds, such as Chihuahuas and Toy Poodles, typically live longer than the largest breeds, such as Great Danes and Irish Wolfhounds (Kraus et al. 2013). Indeed, in dogs, on average, an increase in size by $10 \mathrm{~kg}$ is associated with 6 months to a year of reduced life span (Greer et al. 2007; O’Neill et al. 2013). A similar within-species pattern of exceptionally small genotypes living longer than larger genotypes is found in mice, rats, and horses (Rollo 2002; Miller and Austad 2006).

Just why small breeds or genotypes within species tend to live longer than large breeds or genotypes is not yet fully understood. In mice, a 
K.E. Creevy et al.

broad swath of evidence implicates circulating levels of insulin-like growth factor 1 (IGF-1) as a major contributor (Tatar et al. 2003; Yuan et al. 2009). It turns out that much of the body size variation among dogs is explained by polymorphism in the IGF-1 gene (Sutter et al. 2007; Greer et al. 2011). This hints, but does not yet prove, that like in mice, IGF-1 signaling might also be a major contributor to longevity differences in dogs.

To fully understand the potential for an LD, we need to understand much more than just the genetics of longevity. We need to understand details of the relationship between morbidity and mortality as well as their underlying causes. The companion dog has the potential to contribute greatly in this area because of the wide breed-specific variation in both morbidity and mortality (Bonnett et al. 2005; Egenvall et al. 2005a; Fleming et al. 2011; Kraus et al. 2013).

\section{Mortality}

From a purely demographic perspective, the study of aging is the study of population patterns in age-specific mortality rates. The LD highlights the importance of measuring aging in terms of age-related changes in pathophysiological processes and comorbidities. But, at present, the state-of-the-art focuses on age-specific mortality rates, so let us start with a bit of background and mortality rate analysis. The LD hypothesis assumes that the rate of aging can be slowed. What, precisely, is the rate of aging? Biodemographers commonly focus on the "actuarial rate of aging," which is determined by age-specific mortality rates. Age-specific mortality rates can be intuitively thought of as crude measures of population health. If, say, $1 \%$ of 25 -year-olds die in one population and 10\% of 25-year-olds die in another, then the latter population appears to be substantially less healthy at age 25 , assuming the two populations are in comparable environments. In almost every animal species studied, including humans, adult mortality rates increase with age in a pattern described effectively by the Gompertz model (Gompertz 1825; Promislow 1991; Finch 1990). The Gompertz model describes $\mu_{x}$, the mortality rate at age $x$, using two parameters: a "baseline" mortality rate $\alpha$, usually the lowest mortality rate achieved in adulthood, which occurs around the time of sexual maturity, and an age-dependent parameter $\beta$ :

$$
\mu_{x}=\alpha e^{\beta x} .
$$

If we plot the logarithm of mortality rate against age, the Gompertz curve is a straight line, where the actuarial rate of aging is given by $\beta$, which is the slope of this line. In contrast, $\alpha$ can be thought of as the "intercept" or "elevation" of this line. Intuitively, the Gompertz slope measures the rate of increasing susceptibility to death. A steep slope indicates organisms that deteriorate quickly. A slightly more detailed equation, known as the Siler model, includes a common pattern of initially high juvenile mortality that declines with age (Fig. 1).

From a Gompertzian perspective, longevity in a population can be increased by decreasing $\alpha$, which lowers mortality by an equal proportion at all ages, or by decreasing $\beta$, which reduces the slope, or a combination of both. Biodemographers often focus on decreasing the slope as the only valid metric of a slowed aging rate, because the slope is a measure of how fast things deteriorate. Although this is a valid demographic point, from a health span perspective, it may be preferable to lower $\alpha$. Assuming that the absolute mortality rate at any age is an indicator of population health at that age, then lowering $\alpha$ increases health at all ages by an equivalent amount, whereas decreasing $\beta$ increases the health of the young by a small amount and of the old by a larger amount. Best of all, of course, would be to lower both.

Interestingly, whether genetic or environmental interventions that lengthen life do so by lowering $\alpha$ versus $\beta$ might depend on the species in question. Such interventions in flies mostly lower $\alpha$ (Promislow et al. 1996), whereas in worms they mostly lower $\beta$ (Chen et al. 2007). In mice, it depends on the intervention-some lower one parameter, some the other (de Magalhaes et al. 2005). In humans, most of the increase in life span seen over the past two centuries is caused by a dramatic re- 

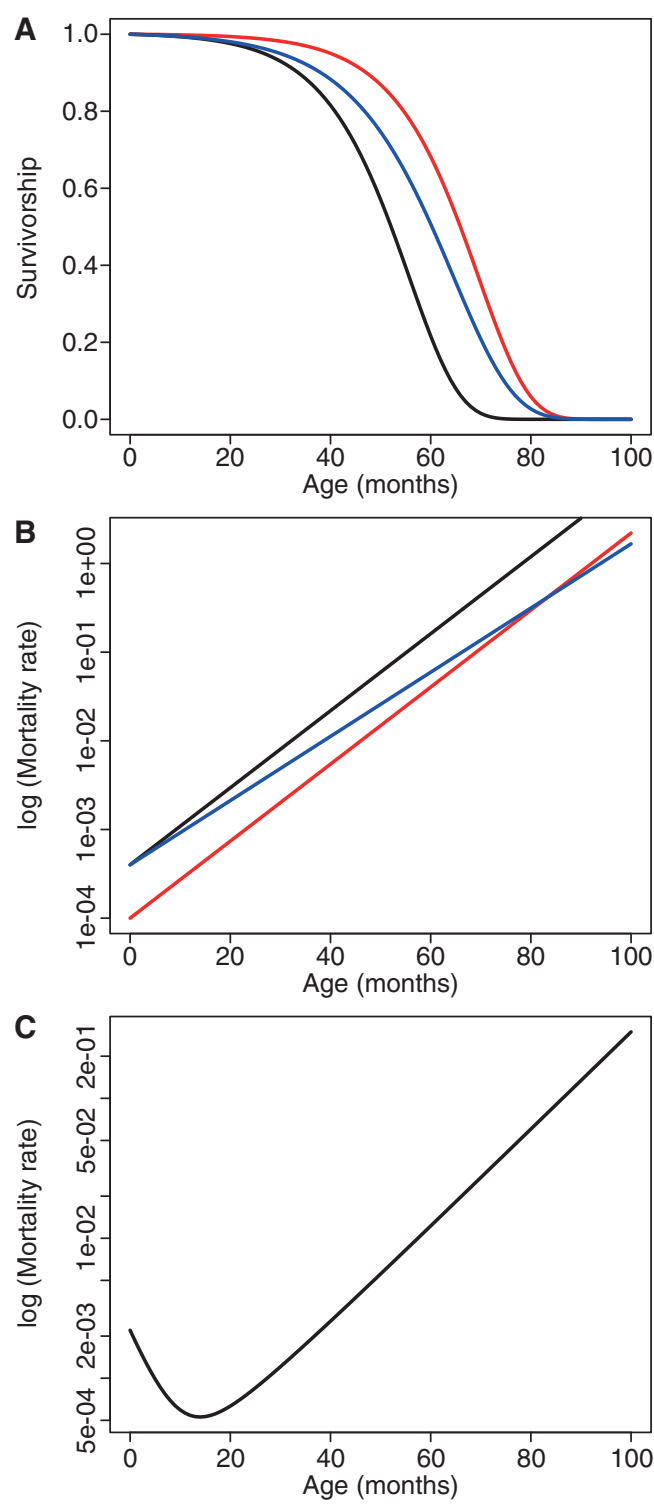

Figure 1. Survivorship curves $(A)$ and age-specific mortality curves $(B)$ for a population following a Gompertz mortality model (Gompertz 1825). According to the Gompertz mortality model, mortality increases exponentially over time, with $\mu_{x}=\alpha e^{\beta x}$, where $\mu_{x}$ is the mortality rate at age $x, \alpha$ is the "baseline" mortality rate, and $\beta$ is the rate of aging. The black line shows standard mortality. Survivorship can be increased (or mortality lowered) either by reducing the baseline mortality (red) or by slowing the rate of aging (blue). Most real-world examples show a much greater reduction in $\alpha$ than in $\beta$. (C) The more realistic Siler mortality model (Siler 1979) is shown, where $\mu_{x}=\alpha_{1} e^{-\beta_{1} x}+\alpha_{2}+\alpha_{3} e^{\beta_{3} x}$.
The Dog as a Model for the Longevity Dividend

duction in early-age mortality, such that we see a large decrease in intercept but an increase in slope (Burger et al. 2012).

Where does the domestic dog fit into this pattern? Is the longevity advantage in small breeds compared with large ones primarily owing to a decrease in the Gompertz slope or intercept? A recent study by one of us suggests that variation in longevity among dog breeds is primarily a function of changing the slope (Kraus et al. 2013). The study relied on data from the Veterinary Medical Database (VMDB) in which exact age at death for the dogs is unknown. Ages at death in the VMDB are placed into bins of varying sizes. For older dogs, one bin comprises animals that died at any time between 10 and 15 years of age, and dogs older than that are simply listed as $15+$. As other data sets with more detailed age-at-death information are compiled, such as VetCompass (O’Neill et al. 2013), we will be able to more accurately determine exactly how rates of aging vary between breeds, assuming that we can come to an agreement on a definition of aging.

Does it matter for the LD whether longevity is enhanced by lowering $\alpha$ versus lowering $\beta$ ? In its original formulation, a goal of the LD is "a modest deceleration in the rate of aging sufficient to delay all aging-related diseases and disorders by about seven years" (Olshansky et al. 2006, p. 32). This would seem to indicate that a lower slope was the goal. On the other hand, 7 years was chosen as a target, because the mortality rate in humans doubles approximately every 7 years. Thus, one could imagine that the investigators envisioned the LD occurring because all agingrelated diseases and disorders were delayed by 7 years, halving the mortality rate at every age-reducing $\alpha$, in other words, to what it had been 7 years earlier in life (Olshansky et al. 2006). Thus, 50-year-olds would, in principle, achieve the health that 43-year-olds formerly enjoyed, and 57 would be the new 50 . This sounds a lot like retarded aging to many of us.

\section{Morbidity}

Any reasonable goal of health-related research should include improving and prolonging 
K.E. Creevy et al.

health. Let us admit that age-specific mortality is a very crude measure of population health. Its related concept, morbidity, describes the consequence of myriad diseases that can significantly affect frailty and health span. A full understanding of the potential for an $\mathrm{LD}$ requires that we understand age-specific risks of mortality and morbidity. Dog breeds vary not only in "when" they die, they also vary tremendously in "why" they die. For the common dog breeds, there is an extensive literature on common ailments and causes of death. For example, Doberman Pinschers have high rates of morbidity and mortality caused by cardiomyopathy, Dachshunds suffer from intervertebral disc disease, and Miniature Schnauzers develop diabetes more often than other common breeds (Hess et al. 2000; Wess et al. 2010). And even for rarer breeds, we can identify highly specific "morbidity profiles" for each breed from large health databases that provide sufficient study sample sizes (Fleming et al. 2011).

Health is not simply delaying the onset or slowing the progression of fatal or potentially fatal diseases. As Bellows et al. (2015) make clear in a recent comprehensive review of healthy aging, many nonfatal maladies of aging, such as joint pain, hearing and vision loss, and muscle weakness, are common in aging dogs. Of particular relevance to this review, we find that just as in humans there are many fatal and nonfatal maladies in dogs that increase with age. As Waters (2011) has noted, what we can measure in humans, we can measure in dogs. So identifying and exploring these maladies in dogs should be as successful as it is for humans. This illustrates the possibility to examine whether slowing aging in dogs might delay the onset, or reduce the frequency, of cancer, heart disease, and diabetes as well as reduce joint pain and maintain mobility and sensory function.

As we noted previously, body size accounts for much of the variation in the timing of death across dog breeds. The same is true for the onset of many age-related diseases or conditions. Cancer incidence increases dramatically with age in all breeds, but it occurs later in smaller breeds compared with large ones (Bonnett et al. 2005; Fleming et al. 2011). The same is true of cataracts (Urfer et al. 2011). Moving forward, comprehensive analyses of LD in dogs should include body size as a key covariate.

\section{DOES WHAT WE KNOW ABOUT DOG AGING SUPPORT THE LD HYPOTHESIS?}

To set the stage for LD, Olshansky et al. (2006) first noted that even if one were to eliminate the diseases that top the list as causes of mortality in human populations, one would see only a modest life span extension. To determine whether the same is true in dogs, we explored five causes of mortality in the VetCompass data set.

The VetCompass Programme is a novel research initiative that began in the United Kingdom and is now being extended worldwide. The philosophy is to capture the cumulative veterinary clinical experience via a large-scale collection of veterinary electronic clinical records that are made accessible for research. The VetCompass Programme (see www.rvc.ac.uk/ vetcompass) was developed at the Royal Veterinary College in conjunction with the University of Sydney with the aim of sharing, analyzing, and disseminating veterinary clinical information to develop an evidence database on the health and welfare of companion animals. VetCompass began collecting data in 2009 and, by 2014, almost 500 veterinary clinics in the United Kingdom have already shared data on more than two million dogs, which offer detailed health experiences covering up to 5 years of life for individual dogs. To date, these data have supported 12 peer-reviewed publications on dogs that cover general longevity and morbidity as well as specific disorders.

The most common cause of death, cancer, kills one in every six dogs in the VetCompass database. And yet, even if we were to eliminate all deaths caused by cancer, the increase in life span is modest (Fig. 2A). In contrast, if we slow aging by $10 \%$, a relatively small amount compared with what we can already accomplish in the laboratory in yeast, worms, flies, and mice, we see a similar increase in life span (Fig. 2B,C).

But companion dogs can also allow us to think more carefully about LD. Recall that the LD hypothesis assumes that ameliorating the 

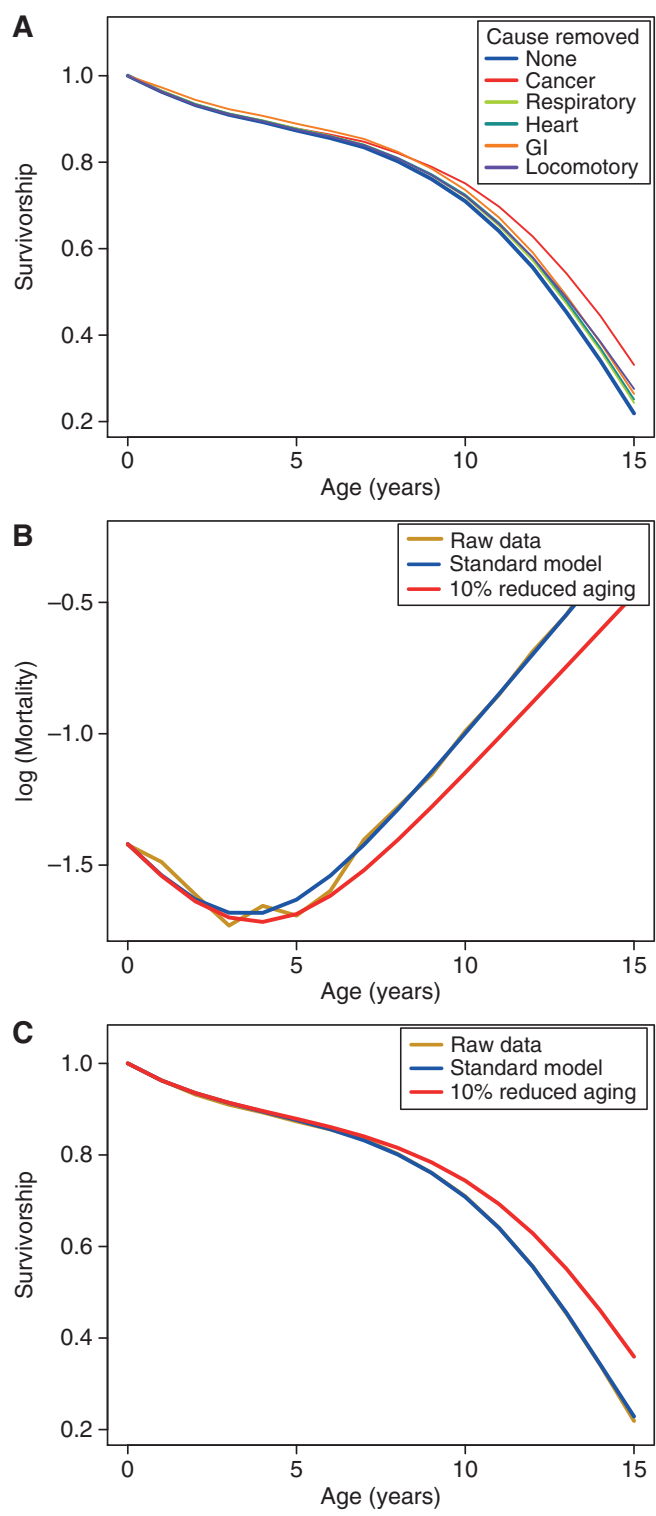

Figure 2. Longevity dividend (LD) calculations for dogs from the VetCompass database (O'Neill et al. 2013). (A) Survival curves for all dogs (blue line) and hypothetical survival curves for populations in which a single cause of mortality is omitted (see text for details). All-cause mortality curves $(B)$ and survivorship curves $(C)$ are shown for actual data (yellow), and based on fitted mortality curves using the Siler model (Siler 1979), $\mu_{x}=\alpha_{1} e^{-\beta_{1} x}+\alpha_{2}+\alpha_{3} e^{\beta_{3} x}$, for all mortality (blue) and for reduced aging rate, where $\beta_{3}$ is the rate of aging and has been reduced by $10 \%$ (red).
The Dog as a Model for the Longevity Dividend

underlying processes of aging will (1) delay the onset and/or slow the progression of aging-related diseases and conditions, and (2) compress the period of ill health at the end of life. We have already seen that many smaller dog breeds live substantially longer than the largest dog breeds, and these small breeds also develop many agingrelated diseases at later ages than large breeds. This is clearly consistent with the LD hypothesis. However, we currently have no evidence about the relative length of illness or morbidity late in life among the various breeds. A study to determine whether there is morbidity compression among smaller breeds would be a valuable addition to this field of knowledge.

One complication in interpreting the LD perspective is that the prevalence of some diseases, such as infectious diseases, decreases with age in dogs (Fleming et al. 2011). Is it possible that retarding the rate of aging might prolong the period of susceptibility to diseases most likely to strike early in life? For instance, parvoviral enteritis is an often-fatal disease that preferentially strikes young dogs. There is now an effective vaccine, but before the widespread use of that vaccine, most cases of parvoviral enteritis struck dogs $<6$ months of age and very few cases occurred in dogs older than 2 years (Mason et al. 1987). This situation is complicated to interpret for several reasons. First, because the most susceptible dogs were not sexually mature and therefore not yet actually aging according to most interpretations, its relevance to the LD hypothesis is not obvious. Interventions thought to slow aging are unlikely to be imposed before sexual maturity. Second, this increased susceptibility may be because of an immature, prepubescent immune system, so this is also difficult to reconcile with the LD hypothesis. On the other hand, interventions that have successfully lengthened life in laboratory animals, such as DR or rapamycin, have been associated with some signs of immune suppression (Gardner 2005; Nikolich-Zugich and Messaoudi 2005; Ritz et al. 2008; Goldberg et al. 2015), potentially interpretable as a return to an immature immune system. Thus, slowing at least some aspects of aging and extending "potential" longevity could potentially increase some diseases 
K.E. Creevy et al.

early in life. In thinking about the LD then, it becomes imperative that we have a comprehensive understanding not only of the effects of age on different pathophysiological systems and processes, but also how specific interventions influence these systems and processes. A necessary step in the development of interventions that may lead to an LD must include assessment of the period in the life span that the intervention is likely to be most effective at reducing both early- and late-life diseases and debilities. As a case in point, which has been extensively studied in dogs, we now turn to the effects of sterilization on longevity and patterns of disease.

\section{Reproduction, Sterility, and the LD}

Companion animals in the United States are often surgically sterilized at the request of their owners to prevent unwanted reproduction or to limit unwanted behaviors. Surgical sterilization has been associated with longer life in a number of large studies on multibreed/mixed breed dogs (Bronson 1982; Michell 1999; Hoffman et al. 2013; O’Neill et al. 2013) as well as in human males (Hamilton and Mestler 1969; Min et al. 2012). Consequently, a large and accessible population of dogs is available to assess the health impact of this longevity-associated procedure.

The most comprehensive assessment of how surgical sterilization affects the causes of death in dogs is that by Hoffman et al. (2013). In that study of more than 40,000 companion dogs in the VMDB known to have died in veterinary teaching hospitals, sterilization was associated with a $19 \%$ increase in longevity (14\% in males, $26 \%$ in females). The VMDB is limited to animals presented to tertiary care hospitals, which could lead to numerous biases (Fleming et al. 2011; Hoffman et al. 2013). However, a contemporaneous study in the United Kingdom using VetCompass data, based on dogs presented to primary care veterinary clinics, reported similar longevity benefits of neutering, at least in females (O’Neill et al. 2013, 2015). Hoffman et al. found-as have a number of previous studies - a higher prevalence of death by cancer in sterilized compared with intact dogs and also a greater prevalence of deaths owing to immune-mediated diseases (see also Hart et al. 2014). On the other hand, sterilized dogs were less likely to die from infectious diseases, trauma, vascular, and degenerative diseases compared with intact dogs. Although we would expect longer-lived (sterilized) dogs to die from diseases that are more prevalent at late age (e.g., cancer), even after controlling for this potential confound statistically, these patterns were still evident. Interestingly, in a study of sterilized versus intact people, deaths from infectious diseases were also reduced in the sterilized group (Hamilton and Mestler 1969).

The "timing" of sterilization vis-à-vis an animal's life history can also be important in the development of diseases. As sterilization removes an entire organ system and its endocrine axis, it would stand to reason that whether that system is removed before adulthood, when the endocrine axis becomes particularly active, might be expected to have large effects throughout life. In the United States, dogs are traditionally sterilized around the time of sexual maturity or shortly thereafter, but debate exists over the relative health costs and benefits of pre-versus postmaturation sterilization. As a gross generalization, dogs are often considered to be sexually mature by 1 year of age. This generalization masks a great deal of breed and individual variation, however, as sexual maturity in dogs varies according to body size. Small dogs can enter puberty as early as 5 to 6 months and some large dogs as late as 2 years of age (Rice 2008).

A number of studies have examined the effects of the timing of sterilization on subsequent health and/or behavior. One of these found that in dogs adopted from animal shelters, animals categorized as having been sterilized at less than 6 months of age (median $=2.5$ months) were more likely to contract parvoviral enteritis than dogs sterilized later (median $=1$ year) (Howe et al. 2001). However, this result is confounded by the fact that parvoviral enteritis is more common in puppies compared with adults as noted before. Breed status of the animals was not identified in this study and the median length of follow-up was only 4 years after sterilization; 
therefore, later-life health problems could not be ascertained. A much larger study - also of dogs adopted from shelters, also without breed identification, also with $\sim 4$ years of follow-upfound that early ( $<5.5$ months) sterilization increased the prevalence of hip dysplasia and, in females only, was also associated with more incontinence and cystitis (Spain et al. 2004). It should be emphasized that these studies only examined the variable of age at sterilization and not the variable of sterilization itself; there were no intact animals for comparison.

The timing of sterilization and its comparison with nonsterilized dogs has also been examined in several studies. Specifically, a large study of Golden Retrievers, which are particularly predisposed to cancer (Fleming et al. 2011), found that sterilization itself as well as the timing of sterilization affected the type of cancers later developed (Torres de la Riva et al. 2013). Specifically, sterilization at any age increased the prevalence of mast cell tumors in females only, although late-neutered ( $>2$ months) females had a higher prevalence of hemangiosarcoma than either intact or early-neutered females. Early neutering of males increased the rate of lymphosarcoma relative to either intact or late-neutered males. Early neutering also increased the rate of skeletal abnormalities, such as hip dysplasia and cranial cruciate ligament tears.

A study of Rottweilers has examined how the status and timing of sterilization affects longevity and cause of death (Waters et al. 2009). Like some other studies, this one found that female dogs lived longer than males (Egenvall et al. 2005b). Unlike a number of multibreed, multisex studies, however, this one found that sterilizing female Rottweilers before 4.5 years of age shortened life. Moreover, females in a group of animals chosen for the study because of their exceptional longevity ( $>30 \%$ greater than breed longevity average) were more likely than females of average life span to have retained their ovaries for more than 4 years. The longest-lived dogs were also at a considerably reduced risk of dying from cancers. A conclusion from this study then could be that exposure to ovarian hormones has a generally beneficial effect on longevity. As the results of this study
The Dog as a Model for the Longevity Dividend

are at variance with multiple other longevity studies that did not include the timing of sterilization, it is worth considering some possible reasons for the difference. Most obviously, this result could be a particular feature of the Rottweiler breed. Second, this result could be a function of the selection of the study population. Among the usual longevity dogs in this group, $73 \%$ died of cancer. This is a substantially higher incidence of fatal cancers than reported for dogs as a whole (Bronson 1982) or for other populations of Rottweilers (Hoffman et al. 2013). Third, the timing of sterilization was very different in the usual longevity group ( $>40 \%$ sterilized within the first year of life) and the exceptional longevity group (40\% sterilized by about 3 years of age) suggesting that the dogs may have been treated quite differently in terms of medical attention or weight maintenance or the quality and amount of their food. Note that exceptional longevity dogs were on average $5 \mathrm{~kg}$ lighter than usual longevity dogs, died considerably less often of cancer, including osteosarcoma to which Rottweilers are particularly prone, and had a much lower incidence of gastrointestinal deaths among noncancer deaths than usual longevity dogs. Provocatively, there were many more deaths from "frailty," in the exceptionally long-lived dogs. Frailty in this case was defined as a "combination of agerelated disabilities, including deficits in mobility, cognition, hearing, eyesight, and inability to maintain body weight" (Waters et al. 2009, p. 753). This description does not seem particularly compatible with the LD. At present, it is not possible to choose among these various interpretations of the Rottweiler study.

Longevity is a composite variable of myriad events and susceptibilities. Although the net effect of sterilization is to increase overall life span in dogs, this longevity comes about through complex effects on causes of death in specific dog genotypes. Can health span become the tool by which we measure the overall costs and benefits of an intervention that decreases the risk of some causes of mortality at the expense of increasing the rate of other causes? Can we determine that the causes of death or length of debility that become more prevalent in longer- 
K.E. Creevy et al.

lived individuals detract from the value of those added months or years of life? The ability to evaluate dogs as individuals with respect to physiological and biochemical parameters, cognitive function, activity level, task performance, longevity, genetics, pharmacogenomics, and so on, suggests that dogs could be a powerful model for the creation of a standardized assessment of health span. Moreover, companion dogs offer the potential to identify interventions that could improve health span, providing an LD for both companion animals and their owners.

\section{Delayers, Escapers, Survivors, and the LD}

In a previous section, we highlighted age-related changes in various causes of death, including cancer, in a large population of dogs. As in humans, mice, rats, and virtually every other vertebrate species, dogs generally show a dramatic increase in risk of cancer with age (Finch 1990). But there is a subtler pattern in the cancer data that recapitulates another pattern seen in humans. In studies of human centenarians - those paragons of successful aging - researchers have suggested that three routes to exceptional longevity can be observed. There are people who live long despite being diagnosed with ageassociated illnesses earlier in life, called Survivors; those who escape age-associated illnesses until late in life, called Delayers; and those who seem to escape most age-associated diseases to the very end of life, called Escapers (Evert et al. 2003). A study in Rottweilers suggests that they, too, might include Delayers or Escapers (Cooley et al. 2003). Within a cohort of 345 animals, among the oldest dogs, cancer rates were actually lower than in younger dogs. It is as though the oldest dogs made up a pool of high-quality Escapers that were comparatively immune to cancer. If a young cohort includes dogs of variable quality, these "Escapers" might simply reflect the highest quality individuals that were both likely to live the longest and were inherently resistant to cancer and other diseases.

To determine if this is true in other dog breeds, we examined age-specific patterns of cancer prevalence in the Veterinary Medical Database (see wvdb.org) for a subset of very common breeds. Interestingly, we saw the same pattern in our data set for large-breed, relatively short-lived dogs. To our surprise, however, we did not observe the same pattern in smaller, longer-lived breeds (Fig. 3). This observation could be because of the fact that the highest two age classes in the VMDB $(10-15$ years and 15+ years) represent a large span of age collapsed into only two categories, so might mask underlying age-specific trends in the smaller breeds in which many individuals survive to these ages. Within the VetCompass data, which benefits from offering the precise age at death for all individual dogs, we observe a pattern of all breeds showing decreased rates of cancer in the later ages regardless of size (Fig. 3). However, at least for now, the VetCompass sample sizes for each age class are much smaller than the VMDB, such that late age data is relatively noisy.

From the perspective of the LD, we are left to wonder whether an increase in life expectancy is accompanied by an increase in the frequency of Escapers and/or Delayers, which would, indeed, pay an LD. Alternatively, it could simply mean more Survivor individuals living with, and suffering from, age-related morbidities.

\section{LIMITATIONS OF THE DOG AS A MODEL FOR LD}

The companion dog presents us with a powerful model to understand the genetic and environmental determinants of age-specific morbidity and mortality. However, as a translational model, dogs do have some important limitations. First, although there is extensive overlap between causes of death in humans and dogs (e.g., Waters 2011), there are also many differences. For example, the spectrum of cancers that is common in dogs includes some cancers that are rare in humans (e.g., hemangiosarcoma, mast cell tumor), although some are relatively common in children (e.g., osteosarcoma).

Second, euthanasia is a common end-oflife decision in dogs, in contrast with humans. A longevity study of dogs in the United Kingdom identified that euthanasia accounted for $86 \%$ of deaths, while only $14 \%$ of deaths were unassisted (O'Neill et al. 2013). This means 

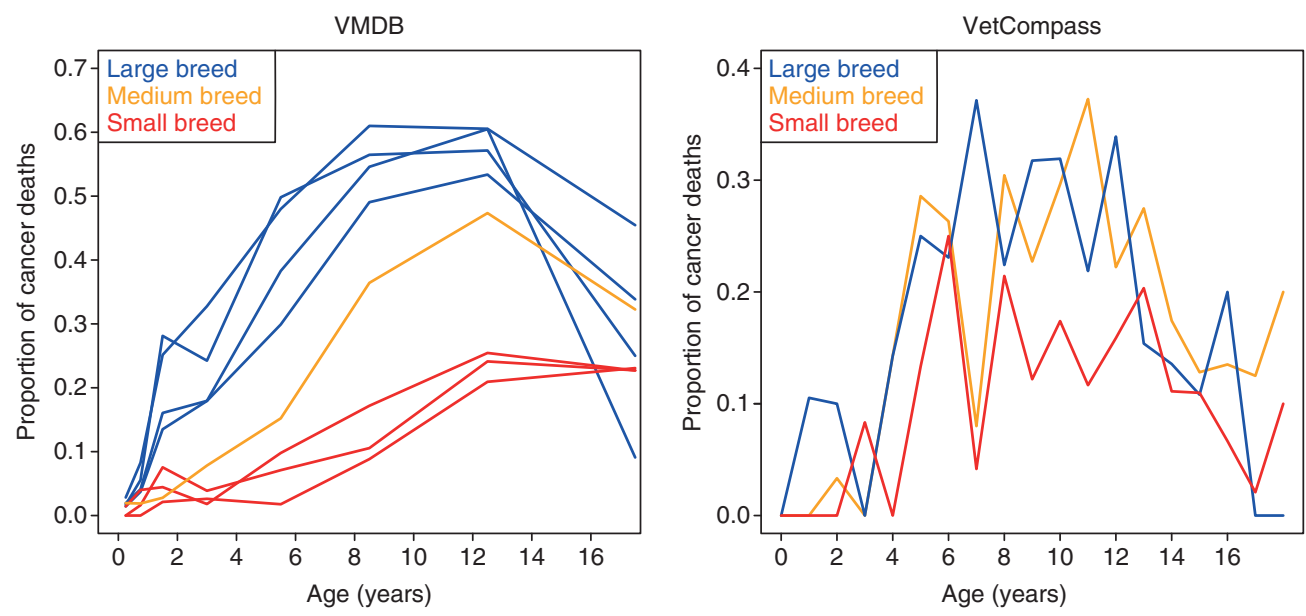

Figure 3. Proportion of dogs dying of cancer for specific breeds. (Left) Veterinary Medical Database (VMDB) data. (Right) VetCompass data. Blue lines indicate large breed dogs (VMDB: Labrador Retriever, Golden Retriever, Rottweiler, and Boxer; VetCompass: Labrador Retriever, Golden Retriever, Rottweiler, and German Shepherd). Orange line indicates medium breed dogs (VMDB: Beagle; VetCompass: Border Collie, American Staffordshire Terrier, English Springer Spaniel, Cocker Spaniel), and the red lines indicate small-breed dogs (VMDB: Miniature Poodle, Dachshund, and Yorkshire Terrier; VetCompass: Cavalier King Charles Spaniel, Jack Russell Terrier, West Highland White Terrier, and Yorkshire Terrier). Notice the trend of high rates of cancer in both data sets with a significant decline in the oldest ages.

that longevity in dogs is not strictly a measure of how long the dogs could live but of how long the dogs' caregivers (owners and veterinarians) believe that they should live. However, the benefit of this situation is that there is usually a defined reason stated to explain the decision to euthanize a dog; $87 \%$ of dogs had a cause of death ascribed (O'Neill et al. 2013). Moreover, although euthanasia tells us nothing directly about the underlying cause of disability, in most cases it likely occurs when morbidity dramatically inhibits the dog's activities of daily living and/or quality of life. As such, it may well be a reasonable measure of the end of "health span" for a dog.

Fries (1980) proposed that we measure functional restriction and disability in studies of morbidity and mortality. Fortunately, in many cases, this is straightforward for a dog: Can it walk, run, climb stairs, etc.? At the same time, other disabilities might be more subtle and harder to detect by both owner and vet, especially when relating to disorders about which dog do not visibly "complain." This might be particularly true of cognitive and psychological problems, which form one of the most common causes of years lived with disability in human populations (Murray et al. 2012).

\section{CONCLUSIONS}

The question remains: If we ameliorate diseases of old age in dogs through extraordinary means, will we prolong the absolute and relative life period of frailty and disability? As is clear from this collection, the answers to this question are far from simple. What is meant by "extraordinary means" depends on the temporal, medical, and species context. A century ago, dogs and humans alike could die from sepsis resulting from a tooth abscess. Today, advanced dental care and antibiotics have dramatically reduced those risks of morbidity and mortality. However, although the lifesaving effects of the antibiotics might be dramatic, the treatment is unlikely to have a noticeable effect on subsequent frailty or disability.

Even when the treatment would seem to qualify as "extraordinary means," the effects on frailty might depend on the details of the 
K.E. Creevy et al.

disease. A dog with mitral valve disease can be spared from heart failure by a few daily pills. And while the dog is running around and eating his pills in bits of food with glee, it is hard to think of his condition as a "disability." On the other hand, if that dog were to progress to heart failure, then keeping him alive with intravenous drugs and an oxygen cage would seem to be extending frailty. Unless, of course, he goes back to running around and eating everything in sight the next day (which is not uncommon). The harder question to answer here is whether a period of poor health has been extended or whether the animal has been returned to a state of good health.

Of course, the dog might not go back to his normal activity or normal appetite ever again, instead teetering in a state of near failure for days or weeks until he finally succumbs or his owners elect for euthanasia. Here we might well say that medical intervention had extended a period of poor health. The dog lived longer, but his state of health in that time would meet no one's definition of health span.

In recent years, the companion dog has emerged as an excellent model to improve our understanding of the determinants of age-specific morbidity and mortality. For centuries, in the course of selecting dogs with specifically defined traits, breeders have unintentionally created lineages that vary considerably in life span and health. We are just now beginning to collect detailed data on age-specific morbidity and mortality across breeds and environments. As we do so, we will have an unprecedented opportunity to explore and better understand the limitations and potential of the LD. Especially in light of the fact that those same treatments (drugs, diagnostic tests, and surgical procedures) used to care for dogs are often also used in humans, there is a critical need to answer the questions posed here. Answers to these important questions will require a concerted and collaborative effort involving not only veterinarians and biodemographers, but also medical ethicists. We are confident that in the coming years, the well-loved canine species will allow us to answer many of the questions posed throughout the literature.

\section{REFERENCES}

Adams VJ, Evans KM, Sampson J, Wood JL. 2010. Methods and mortality results of a health survey of purebred dogs in the UK. J Small Anim Pract 51: 512-24.

Austad SN. 2014. The evolutionary basis of aging. In Molecular and cellular biology of aging (ed. Vijg JC, Lithgow G). Gerontological Society of America, Washington, DC.

Austad SN, Fischer KE. 1991. Mammalian aging, metabolism, and ecology: Evidence from the bats and marsupials. J Gerontol 46: B47-B53.

Bansal A, Zhu LJ, Yen K, Tissenbaum HA. 2015. Uncoupling lifespan and healthspan in Caenorhabditis elegans longevity mutants. Proc Natl Acad Sci 112: E277-E286.

Bellows J, Colitz CM, Daristotle L, Ingram DK, Lepine A, Marks SL, Sanderson SL, Tomlinson J, Zhang J. 2015. Defining healthy aging in older dogs and differentiating healthy aging from disease. J Am Vet Med Assoc 246: 77-89.

Bonnett BN, Egenvall A, Hedhammar A, Olson P. 2005. Mortality in over 350,000 insured Swedish dogs from 1995-2000. I: Breed-, gender-, age- and cause-specific rates. Acta Vet Scand 46: 105-120.

Bronson RT. 1982. Variation in age at death of dogs of different sexes and breeds. Am J Vet Res 43: 2057-2059.

Burger JM, Promislow DEL. 2006. Are functional and demographic senescence genetically independent? Exp Gerontol 41: 1108-1116.

Burger O, Baudisch A, Vaupel JW. 2012. Human mortality improvement in evolutionary context. Proc Natl Acad Sci 109: 18210-18214.

Chang S. 2005. Modeling aging and cancer in the telomerase knockout mouse. Mutat Res 576: 39-53.

Chen J, Senturk D, Wang JL, Muller HG, Carey JR, Caswell H, Caswell-Chen EP. 2007. A demographic analysis of the fitness cost of extended longevity in Caenorhabditis elegans. J Gerontol A Biol Sci Med Sci 62: 126-135.

Cooley DM, Schlittler DL, Glickman LT, Hayek M, Waters DJ. 2003. Exceptional longevity in pet dogs is accompanied by cancer resistance and delayed onset of major diseases. J Gerontol A Biol Sci Med Sci 58: B1078-B1084.

Crimmins EM, Beltran-Sanchez H. 2011. Mortality and morbidity trends: Is there compression of morbidity? J Gerontol B Psychol Sci Soc Sci 66: 75-86.

de Magalhaes JP, Cabral JA, Magalhaes D. 2005. The influence of genes on the aging process of mice: A statistical assessment of the genetics of aging. Genetics 169: 265274.

Egenvall A, Bonnett BN, Hedhammar A, Olson P. 2005a. Mortality in over 350,000 insured Swedish dogs from 1995-2000. II: Breed-specific age and survival patterns and relative risk for causes of death. Acta Vet Scand 46: $121-136$.

Egenvall A, Penell JC, Bonnett BN, Olson P, Pringle J. 2005b. Morbidity of Swedish horses insured for veterinary care between 1997 and 2000: Variations with age, sex, breed and location. Vet Rec 157: 436-443.

Evert J, Lawler E, Bogan H, Perls T. 2003. Morbidity profiles of centenarians: Survivors, delayers, and escapers. J Gerontol A Biol Sci Med Sci 58: 232-237. 
The Dog as a Model for the Longevity Dividend

Finch CE. 1990. Longevity, senescence, and the genome. University of Chicago Press, Chicago.

Fleming JM, Creevy KE, Promislow DE. 2011. Mortality in North American dogs from 1984 to 2004: An investigation into age-, size-, and breed-related causes of death. $J$ Vet Intern Med 25: 187-198.

Freedman VA, Spillman BC, Andreski PM, Cornman JC, Crimmins EM, Kramarow E, Lubitz J, Martin LG, Merkin SS, Schoeni RF, et al. 2013. Trends in late-life activity limitations in the United States: An update from five national surveys. Demography 50: 661-671.

Freedman AH, Gronau I, Schweizer RM, Ortega-Del Vecchyo D, Han E, Silva PM, Galaverni M, Fan Z, Marx P, Lorente-Galdos B, et al. 2014. Genome sequencing highlights the dynamic early history of dogs. PLoS Genet 10: e1004016.

Fries JF. 1980. Aging, natural death, and the compression of morbidity. N Engl J Med 303: 130-135.

Gardner EM. 2005. Caloric restriction decreases survival of aged mice in response to primary influenza infection. J Gerontol A Biol Sci Med Sci 60: 688-694.

Goldberg EL, Romero-Aleshire MJ, Renkema KR, Ventevogel MS, Chew WM, Uhrlaub JL, Smithey MJ, Limesand KH, Sempowski GD, Brooks HL, et al. 2015. Lifespanextending caloric restriction or mTOR inhibition impair adaptive immunity of old mice by distinct mechanisms. Aging Cell 14: 130-138.

Gompertz B. 1825. On the nature of the function expressive of the law of human mortality and on a new mode of determining life contingencies. Phil Trans $R$ Soc Lond 1825: 513-585.

Greer KA, Canterberry SC, Murphy KE. 2007. Statistical analysis regarding the effects of height and weight on life span of the domestic dog. Res Vet Sci 82: 208-214.

Greer KA, Hughes LM, Masternak MM. 2011. Connecting serum IGF-1, body size, and age in the domestic dog. Age (Dordr) 33: 475-483.

Hamilton JB, Mestler GE. 1969. Mortality and survival: Comparison of eunuchs with intact men in a mentally retarded population. J Gerontol 24: 395-411.

Hart BL, Hart LA, Thigpen AP, Willits NH. 2014. Long-term health effects of neutering dogs: Comparison of Labrador Retrievers with Golden Retrievers. PLoS ONE 9: e102241.

Hess RS, Saunders HM, Van Winkle TJ, Ward CR. 2000. Concurrent disorders in dogs with diabetes mellitus: 221 cases (1993-1998). J Am Vet Med Assoc 217: 11661173.

Hoffman JM, Creevy KE, Promislow DEL. 2013. Reproductive capability is associated with lifespan and cause of death in companion dogs. PLoS ONE 8: e61082.

Howe LM, Slater MR, Boothe HW, Hobson HP, Holcom JL, Spann AC. 2001. Long-term outcome of gonadectomy performed at an early age or traditional age in dogs. $J$ Am Vet Med Assoc 218: 217-221.

Kaminski J, Schulz L, Tomasello M. 2012. How dogs know when communication is intended for them. Dev Sci 15: 222-32.

Kraus C, Pavard S, Promislow DEL. 2013. The size-life span trade-off decomposed: Why large dogs die young. Am Nat 181: 492-505.
Larson G, Karlsson EK, Perri A, Webster MT, Ho SY, Peters J, Stahl PW, Piper PJ, Lingaas F, Fredholm M, et al. 2012. Rethinking dog domestication by integrating genetics, archeology, and biogeography. Proc Natl Acad Sci 109: 8878-8883.

Li Y, Deeb B, Pendergrass W, Wolf N. 1996. Cellular proliferative capacity and life span in small and large dogs. $J$ Gerontol A Biol Sci Med Sci 51: B403-B408.

Mason MJ, Gillett NA, Muggenburg BA. 1987. Clinical, pathological, and epidemiologic aspects of canine parvoviral enteritis in an unvaccinated closed beagle colony1978-1985. J Am Anim Hosp Assoc 23: 183-192.

Michell AR. 1999. Longevity of British breeds of dog and its relationships with sex, size, cardiovascular variables and disease. Vet Rec 145: 625-629.

Miklosi A, Soproni K. 2006. A comparative analysis of animals' understanding of the human pointing gesture. Anim Cogn 9: 81-93.

Miller RA, Austad SN. 2006. Growth and aging: Why do big dogs die young? In Handbook of the biology of aging, 6th ed. (ed. Masoro JE, Austad SN). Academic, San Diego.

Min KJ, Lee CK, Park HN. 2012. The lifespan of Korean eunuchs. Curr Biol 22: R792-R793.

Murray CJ, Vos T, Lozano R, Naghavi M, Flaxman AD, Michaud C, Ezzati M, Shibuya K, Salomon JA, Abdalla S, et al. 2012. Disability-adjusted life years (DALYs) for 291 diseases and injuries in 21 regions, 1990-2010: A systematic analysis for the Global Burden of Disease Study 2010. Lancet 380: 2197-2223.

Nikolich-Zugich J, Messaoudi I. 2005. Mice and flies and monkeys too: Caloric restriction rejuvenates the aging immune system of non-human primates. Exp Gerontol 40: 884-893.

Oeppen J, Vaupel JW. 2002. Demography. Broken limits to life expectancy. Science 296: 1029-1031.

Olshansky SJ, Perry D, Miller RA, Butler RN. 2006. In pursuit of the longevity dividend. The Scientist, March 1, pp. $28-36$.

O'Neill DG, Church DB, McGreevy PD, Thomson PC, Brodbelt DC. 2013. Longevity and mortality of owned dogs in England. Vet J 198: 638-643.

O’Neill DG, Church DB, McGreevy PD, Thomson PC, Brodbelt DC. 2015. Longevity and mortality of cats attending primary care veterinary practices in England. J Feline Med Surg 17: 125-133.

Promislow DEL. 1991. Senescence in natural populations of mammals: A comparative study. Evolution 45: 18691887.

Promislow DEL. 1993. On size and survival: Progress and pitfalls in the allometry of life span. J Gerontol 48: B115B123.

Promislow DEL, Tatar M, Khazaeli AA, Curtsinger JW. 1996. Age-specific patterns of genetic variance in Drosophila melanogaster. I: Mortality. Genetics 143: 839-848.

Reiser K, McGee C, Rucker R, Mcdonald R. 1995. Effects of aging and caloric restriction on extracellular matrix biosynthesis in a model of injury repair in rats. J Gerontol A Biol Sci Med Sci 50A: B40-B47.

Rice D. 2008. The complete book of dog breeding. Barrons, Hauppauge, NY. 


\section{K.E. Creevy et al.}

Ritz BW, Aktan I, Nogusa S, Gardner EM. 2008. Energy restriction impairs natural killer cell function and increases the severity of influenza infection in young adult male C57BL/6 mice. J Nutr 138: 2269-2275.

Rollo CD. 2002. Growth negatively impacts the life span of mammals. Evol Dev 4: 55-61.

Salomon JA, Wang H, Freeman MK, Vos T, Flaxman AD, Lopez AD, Murray CJ. 2012. Healthy life expectancy for 187 countries, 1990-2010: A systematic analysis for the Global Burden Disease Study 2010. Lancet 380: 21442162.

Siler W. 1979. Competing-risk model for animal mortality. Ecology 60: 750-757.

Spain CV, Scarlett JM, Houpt KA. 2004. Long-term risks and benefits of early-age gonadectomy in dogs. $J A m$ Vet Med Assoc 224: 380-387.

Sutter NB, Bustamante CD, Chase K, Gray MM, Zhao K, Zhu L, Padhukasahasram B, Karlins E, Davis S, Jones PG, et al. 2007. A single $I G F 1$ allele is a major determinant of small size in dogs. Science 316: 112-115.

Tatar M, Bartke A, Antebi A. 2003. The endocrine regulation of aging by insulin-like signals. Science 299: 1346-1351.

Thatcher AR, Cheung SL, Horiuchi S, Robine JM. 2010. The compression of deaths above the mode. Demogr Res 22: 505-538.
Torres de la Riva G, Hart BL, Farver TB, Oberbauer AM, Messam LL, Willits N, Hart LA. 2013. Neutering dogs: Effects on joint disorders and cancers in golden retrievers. PLoS ONE 8: e55937.

Urfer SR, Greer K, Wolf NS. 2011. Age-related cataract in dogs: A biomarker for life span and its relation to body size. Age (Dordr) 33: 451-460.

Waters DJ. 2011. Aging research 2011: Exploring the pet dog paradigm. ILAR J 52: 97-105.

Waters DJ, Kengeri SS, Clever B, Booth JA, Maras AH, Schlittler DL, Hayek MG. 2009. Exploring mechanisms of sex differences in longevity: Lifetime ovary exposure and exceptional longevity in dogs. Aging Cell 8: 752-755.

Weindruch R, Walford RL. 1988. The retardation of aging and disease by dietary restriction. Charles C Thomas, Springfield, IL.

Wess G, Schulze A, Butz V, Simak J, Killich M, Keller LJ, Maeurer J, Hartmann K. 2010. Prevalence of dilated cardiomyopathy in Doberman Pinschers in various age groups. J Vet Intern Med 24: 533-538.

Yuan R, Tsaih SW, Petkova SB, De Evsikova CM, Xing S, Marion MA, Bogue MA, Mills KD, Peters LL, Bult CJ, et al. 2009. Aging in inbred strains of mice: Study design and interim report on median lifespans and circulating IGF1 levels. Aging Cell 8: 277-287. 


\section{$\&_{\mathrm{CSH}}^{\infty} \&$ Cold Spring Harbor

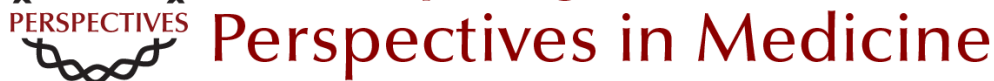

\section{The Companion Dog as a Model for the Longevity Dividend}

Kate E. Creevy, Steven N. Austad, Jessica M. Hoffman, Dan G. O'Neill and Daniel E.L. Promislow

Cold Spring Harb Perspect Med 2016; doi: 10.1101/cshperspect.a026633

Subject Collection Aging

Personalized Financial Planning Using Applied Genetics

S. Jay Olshansky, Bradley Willcox, Kirk Ashburn, et al.

Biological Restraints on Indefinite Survival Jan Vijg and Steven N. Austad

The Role of the National Institute on Aging in the Development of the Field of Geroscience

Felipe Sierra and Ronald A. Kohanski

The Funding Channels of Geroscience Stephanie Lederman

Evolutionary Approaches in Aging Research Melissa Emery Thompson

Interventions for Human Frailty: Physical Activity as a Model

Linda P. Fried

How Research on Human Progeroid and Antigeroid Syndromes Can Contribute to the Longevity Dividend Initiative

Fuki M. Hisama, Junko Oshima and George M. Martin

Articulating the Case for the Longevity Dividend S. Jay Olshansky
Funding Life-Extension Research Mehmood Khan

Influence of Aging Science on Global Wealth

Management Michael Hodin

International Gains to Achieving Healthy

Longevity Andrew Scott, Julian Ashwin, Martin Ellison, et al.

From Life Span to Health Span: Declaring

"Victory" in the Pursuit of Human Longevity S. Jay Olshansky

Crowdfunding and Crowdsourcing of Aging

Science Keith Comito

Inhibition of the Mechanistic Target of Rapamycin (mTOR) -Rapamycin and Beyond Dudley W. Lamming

The Emergence of Geroscience as an

Interdisciplinary Approach to the Enhancement of Health Span and Life Span

Felipe Sierra

The Economic Promise of Delayed Aging Dana Goldman

For additional articles in this collection, see http://perspectivesinmedicine.cshlp.org/cgi/collection/ 